\title{
Computational implications of biophysical diversity and multiple timescales in neurons and synapses for circuit performance
} Julijana Gjorgjieva ${ }^{1}$, Guillaume Drion ${ }^{1,2}$ and Eve Marder ${ }^{1}$

\begin{abstract}
Despite advances in experimental and theoretical neuroscience, we are still trying to identify key biophysical details that are important for characterizing the operation of brain circuits. Biological mechanisms at the level of single neurons and synapses can be combined as 'building blocks' to generate circuit function. We focus on the importance of capturing multiple timescales when describing these intrinsic and synaptic components. Whether inherent in the ionic currents, the neuron's complex morphology, or the neurotransmitter composition of synapses, these multiple timescales prove crucial for capturing the variability and richness of circuit output and enhancing the informationcarrying capacity observed across nervous systems.
\end{abstract}

\footnotetext{
Addresses

${ }^{1}$ Volen Center and Biology Department, Brandeis University, Waltham, MA 02454, United States

${ }^{2}$ Department of Electrical Engineering and Computer Science, University of Liège, Liège B-4000, Belgium
}

Corresponding author: Marder, Eve (marder@brandeis.edu)

Current Opinion in Neurobiology 2016, 37:44-52

This review comes from a themed issue on Neurobiology of behavior Edited by Alla Karpova and Roozbeh Kiani

http://dx.doi.org/10.1016/j.conb.2015.12.008

0959-4388/C) 2015 Elsevier Ltd. All rights reserved.

\section{Introduction}

To what extent can we understand the dynamics of large circuits using biophysical descriptions of single neurons and small subcircuits? In 1989 Getting suggested that the biophysical properties of individual neurons and small circuits could serve as 'building blocks' for a library of biological mechanisms that would aid in understanding all circuits [1]. Getting compiled a partial list of cellular, synaptic and network properties important for neural network operation (Table 1). Many of the features on this list, including intrinsic properties like spike frequency adaptation, post-burst hyperpolarization, and delayed excitation, refer to changes in temporal firing patterns that can last from milliseconds to seconds. Getting also underscored the dramatically different time courses of individual synaptic potentials, such as in the network of interneurons that generate the escape swimming motor program of Tritonia, as well as the ability of a single synaptic connection to mediate several actions with different timescales as seen in multicomponent synapses.

More than 25 years later, we are still struggling to understand which of the myriad of biophysical properties, such as those of Getting's building blocks ('Table 1), are crucial to include in models of brain circuits. Ideally, we should be able to identify a broad array of reusable computational mechanisms that can be combined to generate function and describe circuit dynamics. We suggest that models should capture the relevant timescales of each of the circuit components. These building blocks are often nonlinear; thus, circuit dynamics are the product of a complex spatial and temporal interaction of multiple, nonlinear processes at the cellular and synaptic levels. Therefore, multiple networks that have distinct functions can be realized by using the same constituent building blocks combined in different ways. In this review we highlight recent work that discusses the relevance of biophysical building blocks for circuit dynamics focusing on the role of multiple timescales in the intrinsic and synaptic components of neurons and circuits.

\section{Neuronal intrinsic excitability occurs at multiple timescales}

Elaborate morphologies and diverse ion channels determine the intrinsic excitability of all neurons. To reduce the potential complexity of this high-dimensional space, for many years neuroscientists have been developing strategies to extract the core features of intrinsic neuronal excitability $\left[2^{\bullet}\right]$.

Many studies employ single-compartment models that simplify the neuron's morphology but incorporate specific details of membrane conductances (Figure 1). Choosing the appropriate set of intrinsic conductances depends on the features that the model is aimed at explaining. Some models are constructed by modeling measurements from voltage clamp experiments of all known membrane currents $[3,4]$. Others are more minimalist. For instance, integrate-and-fire or threshold model neurons can be successful in capturing spike initiation dynamics $\left[5^{\bullet}, 6\right]$. But adaptation and history-dependence require additional intrinsic currents [7]. To infer parameter values for these currents from observed membrane potential 


\begin{tabular}{lll}
\hline Building blocks for circuit dynamics by Peter Getting [1] & Connectivity \\
\hline Cellular & Synaptic & Mutual or recurrent inhibition \\
\hline Threshold & Sign & Reciprocal or lateral inhibition \\
f-I relationship & Strength & Recurrent inhibition \\
Spike frequency adapt. & Time course \\
Post-burst hyperpol. & Transmission & Recurrent cyclic inhibition \\
Delayed excitation & Electrical & Parallel excit./inhib. \\
Post-inhibitory rebound & Chemical & \\
Plateau potentials & Release mechanism & \\
Bursting & Graded & \\
Endogenous & Spike & \\
Conditional & Multicomponent PSP & \\
\hline
\end{tabular}

traces, probabilistic frameworks based on statistical inference [8,9] and optimization techniques aimed at minimizing different objective functions have been developed $\left[10^{\circ}\right]$. A recent study has implemented a control theoretic approach to promote alignment between the recorded and model trajectory during the fitting procedure; in addition to fitting synthetically generated data, the procedure also successfully fitted experimental traces $\left[11^{\circ}\right]$.

Approaches for determining appropriate model parameters must overcome the following two challenges: (1) capture the substantial variability observed in experimental measurements of voltage-dependent current densities, ion channel mRNA levels and synaptic connections, suggesting that the space of solutions is highly degenerate and multiple solutions exist for the same output [12$\left.16,17^{\circ}\right]$; (2) achieve robust modulation in spite of variability and degeneracy $\left[18^{\bullet}, 19,20\right]$. Recent studies highlight the importance of building populations of models that capture the variability of parameters seen in experimental measurements [21,22]. Computational database approaches based on parameter exploration of experimentally identified conductances have successfully uncovered multiple and degenerate solutions [23$25,26^{\circ}$. Interesting correlations among intrinsic conductances and neuronal output have been found experimentally at the single neuron level [27], and in computational studies at the circuit level using reduction approaches like principal component analysis to find the interaction of multiple parameters $\left[28^{\circ}\right]$ or simple homeostatic rules operating at the level of the constituent neurons [29 ${ }^{\bullet}$.

Database and model reconstruction approaches have been used to fit ion channel distributions on anatomical reconstructions of known neurons [30-32,33 ${ }^{\circ}$. A recent study showed that, to maintain functional properties along the dendritic tree of a neuron, mechanisms that tune the number of all ion channels collectively are more likely than those that tune the number of individual ion channels - this would not have been seen in single-compartment models $\left[34^{\circ}\right]$. In some instances, such as the implementation of direction selectivity in the mammalian retina, the entire computation relies primarily on the spatial structure of dendrites $\left[35^{\bullet \bullet}\right]$.

Methods have been developed to assess quantitatively the role of biophysical parameter variations in neuronal activity of single compartment models, independent of the neuron model and the set of intrinsic conductances $\left[36^{\bullet \bullet}\right]$. Dynamic input conductances (DICs) are voltagedependent conductance curves that evolve over time, aggregating the activity of all ion channels in the generation of neuronal activity, and are a useful technique to study how diverse ion channels contribute to modulation, robustness and homeostasis in neuronal signaling in different biological systems $\left[36^{\bullet \bullet}, 37^{\bullet}\right]$. Although currently only applicable to single neurons, this method can also include the contribution of synaptic conductances. This should allow the characterization of network dynamics from the analysis of smaller building blocks in more principled ways than large-scale simulations.

\section{Modeling the complexity of biological synapses}

Most models of neuronal networks use simple synapse models that do not capture the full richness of usedependent synaptic dynamics, even when they attempt to represent synaptic learning rules [38-40]. It has been long known that many different neurotransmitters are used in nervous systems, and that the same neurotransmitter can elicit a variety of postsynaptic actions, depending on the properties of the receptors on the postsynaptic membrane (Figure 2a) [41]. Moreover, many neurons contain and release cotransmitters that can act on multiple timescales [42].

In the last several years, a variety of exciting studies of cotransmission in the vertebrate brain are revealing additional features of synapses long-thought simpler than they really are $\left[43-46,47^{\bullet \bullet}, 48^{\bullet}, 49^{\bullet}, 50^{\bullet \bullet}, 51^{\bullet \bullet}\right]$. Midbrain dopaminergic neurons can corelease glutamate and GABA $\left[46,47^{\bullet}, 52\right]$, which have been shown to be regionally heterogeneous $\left[53^{\circ}\right]$ and differentially affected by external perturbations such as cocaine consumption $\left[54^{\circ}\right]$. Neurons can switch their transmitter composition 


\section{Figure 1}

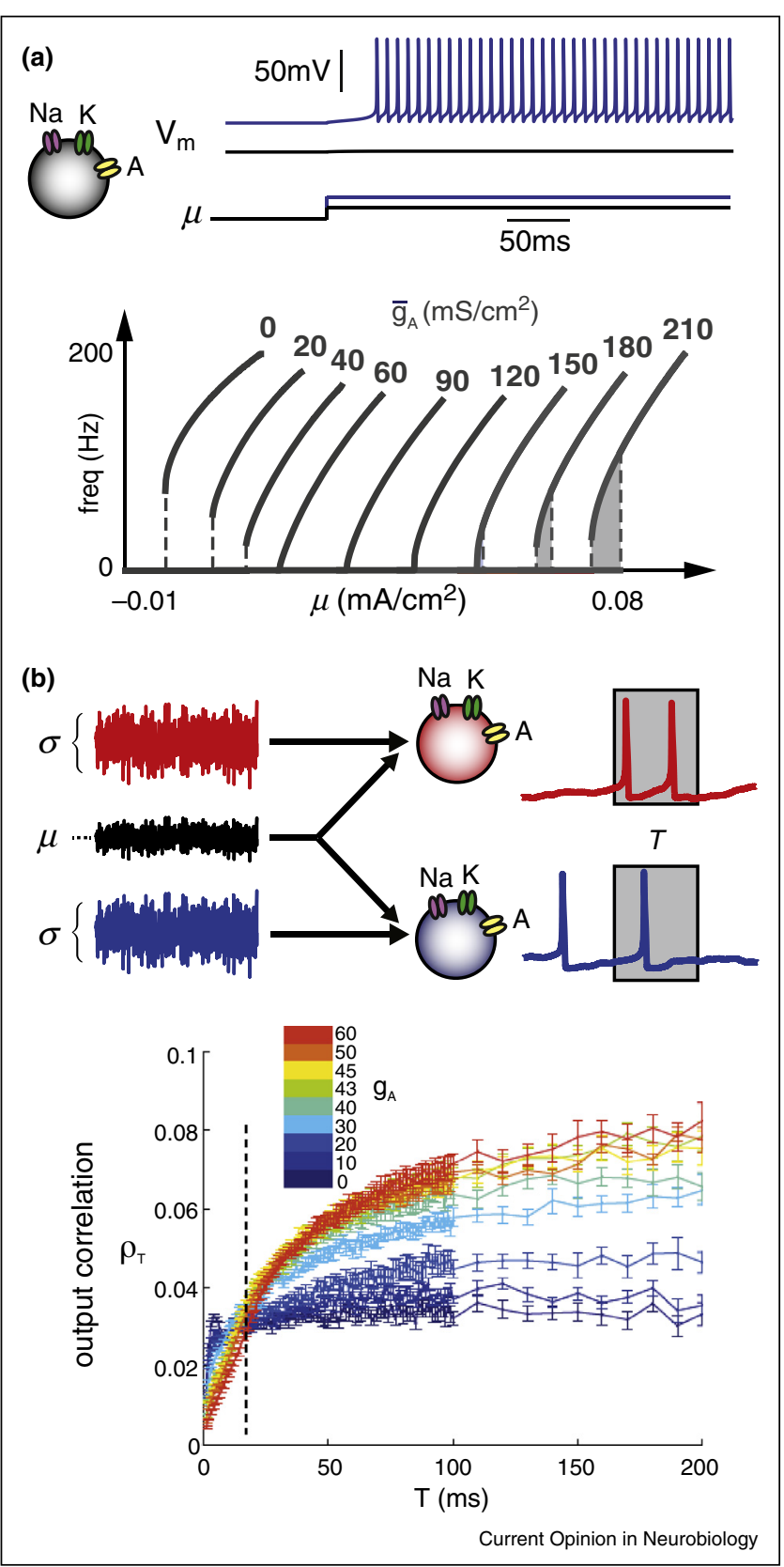

Single neuron biophysics impacts intrinsic properties and correlationbased population coding. (a) Firing rate vs. injected current $(f-l)$ curves, for the Connor-Stevens model [95]. We show $f-l$ curves for a range of $g_{A}$ values yielding a range from type II to type I excitability and then to type $\|^{\star}$ excitability [37]. (b) Top: a microcircuit in which two neurons receive input currents with a common component that represents correlated activity or shared afferents upstream. The mean of the input currents is $\mu$ and each fluctuates with standard deviation $\sigma$. By varying the fraction of the common component (black trace in (b)), relative to the independent components (red and blue traces in (b)), we can control the strength of the input correlation driving the microcircuit. Shared input currents lead to correlated spikes, which are quantified using the correlation coefficient of the two neurons' spike counts counted in time windows of length $T$. Type II neurons with low $g_{\mathrm{A}}$ transfer more correlations at small $T$, while for high $T$ the trend switches (dashed line) with type I neurons with high $g_{\mathrm{A}}$ being able to transfer more correlations [66].
Figure 2

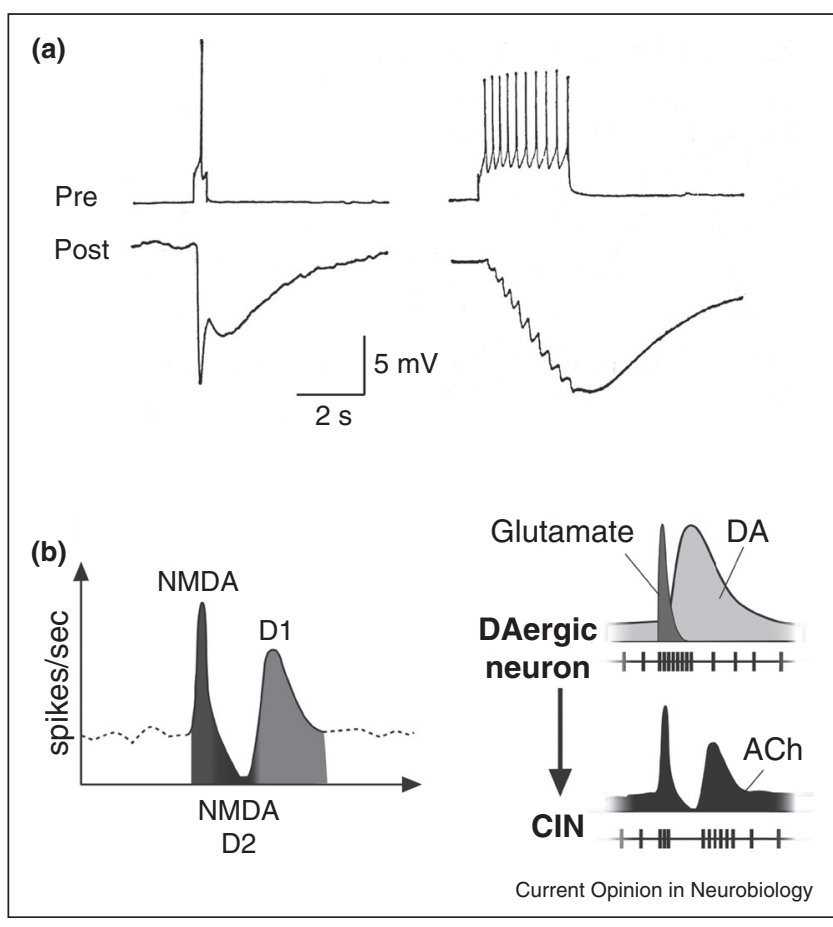

Synaptic transmission occurs at multiple timescales. (a) Left: a twocomponent inhibitory response of medial pleural neuron (in Aplysia) at resting level (Post) to a single presynaptic spike (Pre). Right: typical response of a medial pleural neuron to repeated firing of the presynaptic neuron. A rapid IPSP is associated with each presynaptic spike, whereas the slow IPSP is only evident with repeated firing and is seen as a summated slow wave [41]. (b) Phasic activation of DA controls the three different components of firing in striatal interneurons by coordinated action of glutamate and DA release: glutamate (NMDA) receptor activation evokes an initial burst followed by an afterhyperpolarlization with a firing pause, while DA elicits both a D2-type DAR-dependent firing pause and a late D1-type DAR-dependent burst. DA, dopamine; Ach, acetylcholine; CIN, cholinergic interneuron [58].

over time, both during development and under different physiological conditions in the mature brain leading to changes in behavior $\left[55,56^{\circ}, 57^{\circ}\right]$.

Wieland and colleagues $\left[58^{\bullet \bullet}\right]$ show that the corelease of glutamate and dopamine from midbrain dopamine neurons onto olfactory tubercle cholinergic interneurons induces a triphasic postsynaptic event composed of an early excitation due to glutamate (NMDA) receptor activation, an intermediate inhibition due to dopamine $\mathrm{D} 2$ receptor activation, and a late excitation due to dopamine D1 receptor activation (Figure 2b). Such multicomponent drive underlies the typical response of striatal cholinergic interneurons to relevant sensory stimuli, which can be an initial burst, a firing pause, a late burst, or a combination of the three.

Khalilov and colleagues have recently demonstrated that GABA actions in the immature hippocampal network critically depend on network state [59 $9^{\bullet \bullet}$. Because of 
transient changes in the postsynaptic chloride driving force, GABA currents transiently switch from depolarizing to hyperpolarizing to depolarizing during giant depolarizing potentials, exerting both excitatory and inhibitory roles. This might explain the otherwise contradictory epileptogenic effect of $\mathrm{GABA}_{\mathrm{A}}$ antagonists observed in this network.

Taken together, these recent observations suggest that the multicomponent nature of synaptic potentials is a critical property that strongly shapes neuronal activity at the network level. Because of the richness of cotransmitters and postsynaptic receptors, transient synaptic release can exert both transient and long-lasting effects on postsynaptic neuron excitability. This argues that network models that treat all synapses with a single timescale are likely to be missing important principles of how brain circuits compute.

\section{From single neuron dynamics to circuit function}

Understanding how single neuron and single synapse dynamics alter circuit behavior has classically been studied in both small and large circuits. In the former, it is possible to see directly how one or more biophysical details influence circuit function. In the latter case, it is common to ask how changes in the properties of a population influence circuit function. In both cases, the challenge is to understand the extent to which the circuit's output is influenced either by its architecture or by the properties of its component elements.

Reciprocal inhibition in half-center oscillators has been studied for 100 years [60-63]. In modeling studies, the two constituent neurons and their synapses are usually identical. Experimental studies, however, have incorporated some variable properties of the intrinsic and synaptic components [63,64]. In a recent modeling study, Dethier and colleagues examine the robustness of halfcenter oscillations made from neurons with different subsets of conductances $\left[65^{\circ \bullet}\right]$. They find that a network with low-threshold T-type calcium current has a slow positive feedback at a timescale that endows the network with increased robustness to intrinsic and external perturbations, relative to a network with an $\mathrm{H}$-conductance. This offers opportunities for reliable modulation $\left[65^{\circ}\right]$.

Information and correlation transfer also depend on the constituent neurons' biophysical properties. Small twoneuron populations allow this feature to be studied analytically. For instance, type I neurons (with more A current) transfer correlations over longer timescales $(100 \mathrm{~ms})$, while type II neurons (with less A current) transfer correlations over shorter timescales $(5 \mathrm{~ms})$ (Figure 1) [66,67]. The next challenge would be to take these small circuit motifs and translate them to describing the dynamics of larger networks $\left[68^{\bullet \bullet}\right]$.
How biophysical properties of single neurons impact network function and coding has been addressed in the context of signal propagation through feedforward networks $\left[6^{\bullet \bullet}\right]$. Mease and colleagues have recently identified a change in the ratio of $I_{\mathrm{Na}}$ and $I_{\mathrm{K}}$ in developing mouse cortical neurons that enables these neurons to adaptively scale the gain of their response to the amplitude of the fluctuations they encounter $\left[70^{\circ}\right]$. In a follow-up study, Gjorgjieva and colleagues examined information transmission at different timescales in networks equipped with neurons with different conductance ratios $\left[69^{\circ}\right]$. Independent of the absolute values of the conductances, the networks either became efficient encoders of fast input fluctuations, or gained the ability to transmit slower, population-wide input variations in the network [69*0. This work underscores the significance of simple changes in conductance parameters in governing how neurons represent and propagate information across multiple timescales in networks.

Several experimental and computational studies address the role of diversity in intrinsic properties for how neuronal populations process stimuli and produce robust output. Some recent work examines the possibility that intrinsic properties are tuned to maximize the information of the neurons' response about the stimuli they encode $\left[71,72^{\bullet}, 73^{\bullet \bullet}, 74^{\bullet}, 75\right]$. Such theories of efficient coding, however, thus far apply most directly to sensory populations where there is a clear definition of the stimuli that the neurons represent. A future challenge will be to interpret them in the context of larger circuits where information is integrated from different brain regions and sensory modalities.

\section{How do we know what biophysical details matter for circuit performance?}

We are starting to see increasing attempts to build very large networks of neurons with biophysically "realistic" sets of conductances [76-78]. While aiming for increased biological verisimilitude, such models, even when carefully constructed and supposedly validated, can be as difficult to understand as the biological systems they are meant to represent. What is worse, these models, no matter how carefully constructed, are always 'wrong,' as they fail to contain all the biological machinery that is either unknown or viewed as less fundamental by the investigator. Paradoxically, up to a point, increasing biological realism in large-scale networks probably aids understanding, while past some point, increasing biological realism impedes understanding. At present, it is unclear where the inflection point describing model complexity and increased understanding lies. Used well, with specific questions in mind, large-scale biophysically-realistic models can drive intuition [77,78]. Otherwise, they risk adding mystery and confusion. 
To understand circuit dynamics as a function of their intrinsic and synaptic properties, it is necessary to have a reliable measure of circuit output. In some cases, circuit behavior is clear. Primary sensory circuits like the retina in the visual system and the olfactory bulb in the olfactory system can be described by well-defined input-output relations. For motor circuits, it is relatively easy to quantify circuit performance.

Quantifying circuit dynamics becomes a difficult problem if circuits are degenerate so that understanding the role of any one attribute in circuit function is nontrivial because a manipulation of a single component can have different effects depending on the particular ways that circuit components are combined. Older studies on the Aplysia gill withdrawal reflex showed that variable sets of neurons can participate in the production of a given behavior and that no two trials produce the same pattern [79-81]. Sensory neurons in $C$. elegans exhibit stochastic responses to the repeated presentation of the same sensory stimulus [82]. This variability is present at the level of behavior as well: behavioral variability persists even when responses of sensory neurons are reproducible $\left[83^{\circ \bullet}\right]$. Moreover, the sensitivity to specific odors shows increased variability across individual animals relative to repeated stimulation in one animal, and adaptation of response variability can be observed in multiple trials $\left[83^{\circ}\right]$.

The analysis of small rhythmic circuits can help discover principles in larger circuits. For instance, neurons can switch in and out of different oscillatory subnetworks, or participate in two rhythms at the same time $\left[84^{\circ}\right]$. Computational and experimental studies can help us uncover degenerate mechanisms by which such switching occurs $\left[18^{\bullet}, 84^{\bullet}, 85\right]$. But the main challenge remains: if different neurons are active in multiple trials to repeated presentation of the same stimulus, how do we determine the role of individual biophysical properties in different states of neuronal activity?

In higher brain areas, it can be less than obvious what computations the circuits perform. Parallel results of variable output have been found in recordings of larger networks, such as the place cells in the rodent hippocampus during a virtual navigation task. The place cells exhibit location-specific firing so that their activity is confined with remarkable precision to a cell-specific part of the environment. Despite this spatial precision, the temporal firing pattern is not nearly as reliable $[86,87]$. Variability across individuals is also prevalent in studies of the human cortex, as shown by functional magnetic resonance imaging $\left[88,89^{\circ}\right]$.

\section{Conclusions}

Models of the future will need to capture more explicitly the multiple timescales shaping intrinsic and synaptic excitability. Although we can learn much from small circuits that produce well-defined outputs, the challenge will be to transfer that knowledge to understand the operation of larger brain circuits that integrate information from different sensory modalities and internal states as in the case of behaving animals. Recent studies have underscored the widely variable internal dynamics and responsiveness to external stimuli across different behavioral contexts and brain states [90]. Neuromodulators modulate intrinsic currents and thus control the excitability of cortical neurons as well as the generation of slow oscillations. These modulations occur on fast timescales that cannot be explained with processes like long-term potentiation and depression that change the strength of synaptic connections over many minutes or hours. Thus, to account for neuromodulation and homeostasis on one hand $\left[29^{\circ}, 91,92^{\circ}\right]$, and long-term synaptic plasticity on another $\left[93,94^{\circ}\right]$ it will be necessary to build models of timescales that can account for the activity ranging from milliseconds to hours and days. Biological systems have managed to find mechanisms that allow them to function on many timescales seamlessly, but we are far from understanding the computational principles that allow this to occur.

\section{Conflict of interest}

Nothing declared.

\section{Acknowledgements}

This work was supported by NIH grant NS17813 and the Swartz Foundation. JG holds a Career Award at the Scientific Interface from the Burroughs Wellcome Fund.

\section{References and recommended reading}

Papers of particular interest, published within the period of review, have been highlighted as:

- of special interest

- of special interest

1. Getting PA: Emerging principles governing the operation of neural networks. Annu Rev Neurosci 1989, 12:185-204.

2. Brunel N, Hakim V, Richardson MJE: Single neuron dynamics - $\quad$ and computation. Curr Opin Neurobiol 2014, 25:149-155. This review characterizes neuronal dynamics through input-output relationships beginning with threshold neurons. The paper discusses the implication of incorporating dendritic nonlinearities into single neuron dynamics with a focus on abstract input-output models comprised of linear and nonlinear features.

3. Traub RD, Wong RK, Miles R, Michelson H: A model of a CA3 hippocampal pyramidal neuron incorporating voltage-clamp data on intrinsic conductances. J Neurophysiol 1991, 66:635-650.

4. Buchholtz F, Golowasch J, Epstein IR, Marder E: Mathematical model of an identified stomatogastric ganglion neuron. $J$ Neurophysiol 1992, 67:332-340.

5. Brette $\mathrm{R}$ : What is the most realistic single-compartment model - $\quad$ of spike initiation? PLoS Comput Biol 2015, 11:e1004114. Brette summarizes the main benefit of single-compartment, conductance-based model neurons that ignore the morphological structure of real neurons by focusing on the aspects that a neuron model is meant to achieve: 'simple enough to be useful but realistic enough to be meaningful.' Brette compares Hodgkin-Huxley-style models with multiple conductances and more simplified integrate-and-fire models that generate action potentials upon crossing threshold, and concludes that the latter are more successful in capturing the spiking responses of cortical 
neurons if the goal is to produce the best model for spike initiation.

6. Jolivet R, Rauch A, Luscher H, Gerstner W: Predicting spike timing of neocortical pyramidal neurons by simple threshold models. J Comput Neurosci 2006, 21:35-49.

7. Lundstrom BN, Higgs MH, Spain WJ, Fairhall AL: Fractional differentiation by neocortical pyramidal neurons. Nat Neurosci 2008, 11:1335-1342.

8. Huys QJM, Paninski L: Smoothing of, and parameter estimation from, noisy biophysical recordings. PLOS Comput Biol 2009, 5:e1000379.

9. Lepora NF, Overton PG, Gurney L: Efficient fitting of conductance-based model neurons from somatic current clamp. J Comput Neurosci 2011, 32:1-24.

10. Meliza CD, Kostuk M, Huang $H$, Nogaret A, Margoliash D,

- Abarbanel HDI: Estimating parameters and predicting membrane voltages with conductance-based neuron models. Biol Cybern 2014, 108:1495-1516.

The authors implement previously developed techniques for statistical inference of the dynamics of individual Hodgkin-Huxley-style neurons based on a variational approximation. The technique is applied to biological data from neurons recoded in slice preparation of the zebra finch forebrain nucleus HVC. Using short voltage traces $(1.5 \mathrm{~s})$ the procedure estimates over 70 parameters of the dynamic model such that it can successfully predict responses to novel injected currents. The model fits also make interesting predictions on ion channel distributions for different classes of HCV neurons.

11. Brookings T, Goeritz ML, Marder E: Automatic parameter

- estimation of multicompartmental neuron models via minimization of trace error with control adjustment. $J$ Neurophysiol 2014, 112:2332-2348.

The authors present a control-theoretic approach to fit conductancebased neuron models to intracellular voltage traces from isolated biological neurons. The authors find that their models achieve good spiking accuracy on synthetic data and in data from a stomatogastric ganglion neuron of the crab, Cancer borealis, recorded in response to pink noise.

12. Schulz DJ, Goaillard JM, Marder E: Variable channel expression in identified single and electrically coupled neurons in different animals. Nat Neurosci 2006, 9:356-362.

13. Goaillard J-M, Schulz DJ, Taylor1 AL, Marder E: Functional consequences of animal-to-animal variation in circuit parameters. Nat Neurosci 2009, 12:1424-1430.

14. Roffman RC, Norris BJ, Calabrese RL: Animal-to-animal variability of connection strength in the leech heartbeat central pattern generator. J Neurophysiol 2012, 107:1681-1693.

15. Amendola J, Woodhouse A, Martin-Eauclaire M-F, Goaillard J-M: $\mathrm{Ca}^{2+} / \mathrm{cAMP}$-sensitive covariation of $I_{A}$ and $I_{H}$ voltage dependences tunes rebound firing in dopaminergic neurons. $J$ Neurosci 2012, 32:2166-2181.

16. Golowasch J: Ionic current variability and functional stability in the nervous system. BioScience 2014, 64:570-580.

17. Couton L, Mauss AS, Evers JF, Landgraf M: Development of

- connectivity in a motoneuronal network in Drosophila larvae. Curr Biol 2015, 25:568-576.

This study addresses variability in establishing connectivity in the motor network responsible for the generation of crawling in Drosophila larvae. While different motoneurons form cell-type-specific patterns of connections, there is substantial variability in the number of synapses on motoneuron dendrites. Even at the level of making connections with a common interneuron, different connectivity outcomes can arise. This finding is in contrast to the sensory neurons which form much more stereotyped connections.

18. Rodriguez JC, Blitz DM, Nusbaum MP: Convergent rhythm

- $\quad$ generation from divergent cellular mechanisms. J Neurosci 2013, 33:18047-18064.

The authors beautifully demonstrate that different neuromodulatory inputs can elicit the same pattern in a central pattern generating circuit, the gastric mill rhythm in the crab Cancer borealis, via different rhythmgenerating circuits.

19. Marder E, O'Leary T, Shruti S: Neuromodulation of circuits with variable parameters: single neurons and small circuits revea principles of state-dependent and robust neuromodulation. Annu Rev Neurosci 2014, 37:329-346.
20. Grashow R, Brookings T, Marder E: Reliable neuromodulation from circuits with variable underlying structure. Proc Natl Acad Sci USA 2009, 106:11742-11746.

21. Golowasch J, Goldman MS, Abbott LF, Marder E: Failure of averaging in the construction of a conductance-based neuron model. J Neurophysiol 2002, 87:1129-1131.

22. Marder E, Taylor AL: Multiple models to capture the variability in biological neurons and networks. Nat Neurosci 2011, 14:133138.

23. Goldman MS, Golowasch J, Marder E, Abbott LF: Global structure, robustness, and modulation of neuronal models. $J$ Neurosci 2001, 21:5229-5238.

24. Prinz AA, Bucher D, Marder E: Similar network activity from disparate circuit parameters. Nat Neurosci 2004, 7:1345-1352.

25. Rathour RK, Narayanan R: Inactivating ion channels augment robustness of subthreshold intrinsic response dynamics to parametric variability in hippocampal model neurons. J Physiol 2012, 590.22:5629-5652.

26. Lamb DG, Calabrese RL: Correlated conductance parameters - in leech heart motor neurons contribute to motor pattern formation. PLOS ONE 2013, 8:e79267.

The authors identify relationships between the density of intrinsic conductances and the output patterns of a well-identified motor circuit, the leech heart motor network. They build a population of model networks made up of connected multi-compartment Hodgkin-Huxley-style leech heart motor neurons and examine how sensitive network output is to diverse conductance sets. Strong correlations were found to exist between the strength of many conductances, and the authors characterized the impact that these correlations have on characteristic circuit activity.

27. Zhao S, Golowasch J: Ionic current correlations underlie the global tuning of large numbers of neuronal activity attributes. $J$ Neurosci 2012, 32:13380-13388.

28. Doloc-Mihu A, Calabrese RL: Identifying crucial parameter - correlations maintaining bursting activity. PLoS Comput Biol 2014, 10:e1003678.

Using an existing data-base of half-center oscillator models meant to capture rhythmic activity of the leech heartbeat, the authors look for linear correlations among conductance parameters that maintain functional output. They apply principal component analysis to extract three maximal conductances (leak, persistent sodium and potassium) that correlate linearly for the single neurons but not for the half-centers. Interestingly, they find that the half-center circuits are more robust than the single neurons to changes in the individual conductances.

29. O'Leary T, Williams AH, Franci A, Marder E: Cell types, network - homeostasis, and pathological compensation from a biologically plausible ion channel expression model. Neuron 2014, 82:809-821.

This is a theoretical study that demonstrates the emergence of networklevel homeostasis from the homeostatic regulation of individual neurons using a simple homeostatic rule that aims to maintain the average firing rate of a cell using the calcium activity as a sensor. Using this rule, the authors generate different cell types with cell-specific correlations in the intrinsic conductances as a function of their expression rates. They also tune small central pattern generating circuits where network-level homeostasis is also dependent on the correlations of intrinsic conductances of the individual neurons in the circuit.

30. Achard P, De Schutter E: Complex parameter landscape for a complex neuron model. PLOS Comput Biol 2006, 2:e94.

31. Hendrickson EG, Edgerton JR, Jaeger D: The capabilities and limitations of conductance-based compartmental neuron models with reduced branched or unbranched morphologies and active dendrites. J Comput Neurosci 2011, 30:301-321.

32. Günay C, Sieling FH, Dharmar L, Lin W-H, Wolfram V, Marley R, Baines RA, Prinz AA: Distal spike initiation zone location estimation by morphological simulation of ionic current filtering demonstrated in a novel model of an identified Drosophila motoneuron. PLoS Comput Biol 2015, 11:e1004189.

33. Almog M, Korngreen A: A quantitative description of dendritic - conductances and its application to dendritic excitation in layer 5 pyramidal neurons. J Neurosci 2014, 34:182-196.

This study derives a mechanistic model for the generation and propagation of dendritic spikes in neocortical layer 5 pyramidal neurons by 
deriving heterogeneous dendritic conductance gradients for several voltage-gated channels. The authors use a pharmacological peeling procedure from known channel kinetics and a genetic algorithm to reduce parameter space. The optimized model predicted several experimentally interesting features, including: an ionic mechanism for the generation of dendritic spikes, their interaction with backpropagating action potentials, the ability of the proximal apical dendrite to control the coupling between the axon and the dendrite, and the generation of NMDA spikes in the distal apical tuft.

34. Rathour RK, Narayanan R: Homeostasis of functional maps in

- active dendrites emerges in the absence of individual channelostasis. Proc Natl Acad Sci U S A 2014, 111:E1787E1796.

This modeling study addresses the question of whether maps of functional properties along the dendritic tree of a neuron are achieved and maintained by homeostatic mechanisms at the level of single ion channels or through the collective action of multiple ion channels. Using a global sensitivity analysis on morphologically realistic conductancebased models of hippocampal pyramidal neurons they find that functiona maps emerge from disparate model parameters with weak correlations, arguing for a collective ion channel homeostasis behind the robust emergence of single neuron function.

35. Yonehara K, Farrow K, Ghanem A, Hillier D, Balint K, Teixeira M, •• Jüttner J, Noda M, Neve RL, Conzelmann KK, Roska B: The first stage of cardinal direction selectivity is localized to the dendrites of retinal ganglion cells. Neuron 2013, 79:1078-1085. In this elegant study the authors demonstrate that direction selectivity in a class of ganglion cells selective to motion in one of the four cardinal directions first emerges at the dendrites of these ganglion cells. They also demonstrate that neither the axon terminal of bipolar cells, nor their glutamatergic input to the ganglion cells, are direction selective.

36. Drion G, Franci A, Dethier J, Sepulchre R: Dynamic input - conductances shape neuronal spiking. eNeuro 2015, 2:E0031. This study presents a novel methodological framework to quantify the sensitivity of neuronal activity to changes in ion channel densities. The authors develop the idea of dynamic input conductances, or DICs, that combine the activity of all ion channels in the generation of a firing event (three in a bursting neuron: fast for spike upstroke, slow for spike downstroke and interspike period, and ultraslow for spike adaptation and interburst period). It is demonstrated that the DICs can be measured experimentally in real neurons, and extracted from arbitrary conductance-based models. Finally, the authors demonstrate how the DICs can be used to understand reliable neuromodulation in the presence of intrinsic conductance variability and compensation to different perturbations.

37. Drion G, O'Leary T, Marder E: Ion channel degeneracy enables - robust and tunable neuronal firing rates. Proc Natl Acad Sci U S A 2015, 112:E5361-E5370.

This study examines the role of two specific intrinsic conductances in a single-compartment model in determining the shape of the frequencyinput $(f-l)$ curve, a relationship that describes the firing rate of a single neuron as a function of input strength. The authors first show the paradoxical result that the $f-l$ curve transitions from type II to type I back to type II as the A current density is increased using the dynamic input conductances as a tool. Finally they show that a different set of currents can have opposite effects, an example of degeneracy.

38. Clopath C, Büsing L, Vasilaki E, Gerstner W: Connectivity reflects coding: a model of voltage-based STDP with homeostasis. Nat Neurosci 2010, 13:344-352.

39. Lim S, McKee JL, Woloszyn L, Amit Y, Freedman DJ, Sheinberg DL, Brunel N: Inferring learning rules from distributions of firing rates in cortical neurons. Nat Neurosci $2015,18: 1804-1810$

40. Froemke RC: Plasticity of cortical excitatory-inhibitory balance. Annu Rev Neurosci 2015, 38:195-219.

41. Kehoe J: The physiological role of three acetylcholine receptors in synaptic transmission in Aplysia. J Physiol 1972, 225:147-172

42. Jan YN, Jan LY, Kuffler SW: A peptide as a possible transmitter in sympathetic ganglia of the frog. Proc Natl Acad Sci U S A 1979, 76:1501-1505.

43. Hnasko TS, Edwards $\mathrm{RH}$ : Neurotransmitter co-release: mechanism and physiological role. Annu Rev Physiol 2012, 74:225.
44. Kennedy C: ATP as a cotransmitter in the autonomic nervous system. Auton Neurosci 2015, 191:2-15.

45. Hirasawa H, Betensky RA, Raviola E: Corelease of dopamine and GABA by a retinal dopaminergic neuron. J Neurosci 2012 32:13281-13291

46. Tritsch NX, Ding JB, Sabatini BL: Dopaminergic neurons inhibit striatal output through non-canonical release of GABA. Nature 2012, 490:262-266

47. Tritsch NX, Oh W-J, Gu C, Sabatini BL: Midbrain dopamine

- neurons sustain inhibitory transmission using plasma membrane uptake of GABA, not synthesis. eLife 2014 3:e01936.

Cotransmission of dopamine and GABA in midbrain dopamine neurons is shown to rely on GABA uptake from the extracellular milieu rather than through GABA synthesis, providing a way for the neurons to differentially control GABA release at their numerous presynaptic sites.

48. Saunders A, Granger AJ, Sabatini BL: Corelease of acetylcholine - $\quad$ and GABA from cholinergic forebrain neurons. eLife 2015 , 4:e06412.

The authors show that forebrain cholinergic neurons corelease GABA and acetylcholine in cortical layer 1, resulting in fast, bidirectional synaptic potentials carried by the coactivation of $\mathrm{GABA}_{A}$ and nicotinic acetylcholine receptors at the postsynaptic site.

49. Muschamp JW, Hollander JA, Thompson JL, Voren G,

- Hassinger LC, Onvani S, Kamenecka TM, Borgland SL, Kenny PJ, Carlezon WA: Hypocretin (orexin) facilitates reward by attenuating the antireward effects of its cotransmitter dynorphin in ventral tegmental area. Proc Natl Acad U S A 2014 111:E1648-E1655

This study shows that hypocretin and dynorphin are coexpressed in the same synaptic vesicles in hypothalamic neurons. Hypocretin and dynorphin are shown to have opposite effects on reward behavior, which is then controlled by a balance between the two cotransmitters.

50. Vaaga CE, Borisovska M, Westbrook GL: Dual-transmitter - neurons: functional implications of co-release and cotransmission. Curr Opin Neurobiol 2014, 29:25-32.

This review summarizes recent work on cotransmission in the nervous system.

51. Yu X, Ye Z, Houston CM, Zecharia AY, Ma Y, Zhang Z, Uygun DS

- Parker S, Vyssotski AL, Yustos R, Franks NP, Brickley SG, Wisden W: Wakefulness is governed by GABA and histamine cotransmission. Neuron 2015, 87:164-178.

The authors show that paracrine corelease of GABA and histamine by hypothalamic histaminergic neurons controls wakefulness. Overexcitation from histamine is shown to be regulated by the inhibitory action of GABA, as knocking down the vesicular GABA transporter in mouse histaminergic neurons largely increased the amount of sustained wakefulness.

52. Liu S, Plachez C, Shao Z, Puche A, Shipley MT: Olfactory bulb short axon cell release of GABA and dopamine produces a temporally biphasic inhibition-excitation response in external tufted cells. J Neurosci 2013, 33:2916-2926.

53. Chuhma N, Mingote S, Moore H, Rayport S: Dopamine neurons

•- control striatal cholinergic neurons via regionally heterogeneous dopamine and glutamate signaling. Neuron 2014, 81:901-912.

In this experimental study, midbrain dopamine neurons are shown to exert a regionally heterogeneous effect on striatal cholinergic interneurons, due to regional variation in the relative cotransmission of dopamine and glutamate.

54. Adrover MF, Shin JH, Alvarez VA: Glutamate and dopamine

- transmission from midbrain dopamine neurons share similar release properties but are differentially affected by cocaine. $J$ Neurosci 2014, 34:3183-3192.

An elegant study that points out the differential regulation of cotransmitter release in the dopaminergic system.

55. Milner LD, Landmesser LT: Cholinergic and GABAergic inputs drive patterned spontaneous motoneuron activity before target contact. J Neurosci 1999, 19:3007-3022.

56. Dulcis D, Jamshidi P, Leutgeb S, Spitzer NC: Neurotransmitter - $\quad$ switching in the adult brain regulates behavior. Science 2013 , 340:449-453.

An excellent study that provides a direct link between neurotransmitter 
switching in the mature brain following sensory stimulation and behavior Specifically, the authors studied populations of interneurons in the adult rat hypothalamus, which following exposure to short-day and long-day photoperiods, switch expression between dopamine and somatostatin. Manipulation of the dopaminergic neurons led to behaviors that matched those observed following changes in sensory experience during photoperiods of different length.

57. Spitzer NC: Neurotransmitter switching? No surprise. Neuron - 2015, 86:1131-1144.

A comprehensive review that summarizes recent work on neurotransmitter phenotype plasticity, the process by which the profile of neurotransmitter expression of a given neuron changes over time. This plasticity has been found in neurons across many different brain regions, and both during development and adulthood in response to changing activity. The author begins with evidence for switching in the developing neural crest and in the central nervous system first using culture experiments and then in vivo, and concludes with transmitter switching in the mature nervous system and impact on behavior.

58. Wieland S, Du D, Oswald MJ, Parlato R, Köhr G, Kelsch W: Phasic

-. dopaminergic activity exerts fast control of cholinergic interneuron firing via sequential NMDA, D2, and D1 receptor activation. J Neurosci 2014, 34:11549-11559.

The authors show that corelease of glutamate and dopamine by midbrain dopamine neurons exerts a triphasic action on olfactory tubercle cholinergic interneurons through the sequential activation of NMDA, D2 and D1 receptors.

59. Khalilov I, Minlebaev M, Mukhtarov M, Khazipov R: Dynamic -0 changes from depolarizing to hyperpolarizing GABAergic actions during giant depolarizing potentials in the neonatal rat hippocampus. J Neurosci 2015, 35:12635-12642.

This experimental study points out the potentially large impact of postsynaptic neuron activity on synaptic transmission. The authors show that GABA exerts a depolarizing then hyperpolarizing action during gian depolarizing potential in neonatal rat hippocampus due to a transient inversion of chloride flow through $\mathrm{GABA}_{A}$ receptors. This flow inversion is due to a switch in chloride driving force at the postsynaptic site.

60. Brown TG: The intrinsic factors in the act of progression in the mammal. Proc R Soc Lond Ser B 1911:308-319.

61. Perkel DH, Mulloney B: Motor pattern production in reciprocally inhibitory neurons exhibiting postinhibitory rebound. Science 1974, 185:181-183.

62. Wang XJ, Rinzel J: Alternating and synchronous rhythms in reciprocally inhibitory model neurons. Neural Comp 1992, 4:84 97

63. Sharp AA, Skinner FK, Marder E: Mechanisms of oscillation in dynamic clamp constructed two-cell half-center circuits. $J$ Neurophysiol 1996, 76:867-883.

64. Grashow R, Brookings T, Marder E: Compensation for variable intrinsic neuronal excitability by circuit-synaptic interactions. J Neurosci 2010, 30:9145-9156.

65. Dethier J, Drion G, Franci A, Sepulchre R: A positive feedback at - the cellular level promotes robustness and modulation at the circuit level. J Neurophysiol 2015, 114:2472-2484.

This work examines the influence of single neuron biophysics on the dynamics of half-center oscillators. The authors examine two specific ionic currents, a hyperpolarization-activated cation current and lowthreshold T-type calcium current that can both generate a postinhibitory rebound but differ in that only one of them generates slow positive feedback through its slow activation. This simple feedback mechanism at the cellular level is invaluable for ensuring robustness and modulation at the circuit level.

66. Barreiro A, Thilo EL, Shea-Brown E: A-current and type I/type II transition determine collective spiking from common input. $J$ Neurophysiol 2012, 108:1631-1645.

67. Hong S, Ratté S, Prescott SA, de Schutter E: Single neuron firing properties impact correlation-based population coding. $J$ Neurosci 2012, 32:1413-1428.

68. Ratté S, Hong S, De Schutter E, Prescott SA: Impact of neuronal

- properties on network coding: roles of spike initiation dynamics and robust synchrony transfer. Neuron 2013, 78:758772.

An excellent review addressing different coding schemes at the network level and how they depend on cellular intrinsic properties. For instance, the authors distinguish between two coding schemes, 'synchrony-based' and 'rate-based' coding that depend on whether information is transmitted down-stream in networks through the synchronous firing of many neurons or through their average firing rate. They discuss two extremes of single neuron computation, integrators and coincidence-detectors determined through intrinsic properties, such as their frequency-current relationships and the linear filters by which they process temporal stimuli. They conclude that pyramidal neurons operate in the intermediate regime between these two types, and thus likely implement a multiplex coding scheme, combining synchrony with firing rate codes.

69. Gjorgjieva J, Mease RA, Moody WJ, Fairhall AL: Intrinsic neuronal

- properties switch the mode of information transmission in networks. PLoS Comput Biol 2014, 10:e1003962.

This work builds on Mease et al. (2013) that identified the gain-scaling adaptation property observed experimentally in single neurons during cortical development, and examines the computational implications for information transmission in networks comprised of neurons with different abilities to gain-scale. Since the gain-scaling ability is related to the balance of the two main spike-generating currents, sodium and potassium, the authors build two types of networks with different ratios of these currents. The different single neuron properties of the two types allow the network to transmit information at either fast or slow timescales. The authors relate this to the natural state of the cortex at two developmenta ages, slow wave propagation of spontaneous events in early development, and fast local efficient computations at later ages when sensory stimuli are processed.

70. Mease RA, Famulare M, Gjorgjieva J, Moody WJ, Fairhall AL:

- Emergence of efficient encoding by single neurons in the developing cortex. J Neurosci 2013, 33:12154-12170.

This work combines experimental and theoretical modeling to characterize a single neuron adaptation property that emerges in developing cortical neurons days after birth. The authors build phenomenological linear-nonlinear models to fast fluctuating random input of variable magnitude to describe gain-scaling, which refers to the ability of the neurons to scale their nonlinear input-output relation to the scale of their input. Interestingly, they relate the emergence of this property to the maturation of the two main spike-generating currents, sodium and potassium, and build Hodgkin-Huxley style models as well as reduced exponential integrate-and-fire models to reproduce gain-scaling in model neurons.

71. Padmanabhan K, Urban NN: Intrinsic biophysical diversity decorrelates neuronal firing while increasing information content. Nat Neurosci 2010, 13:1276-1282.

72. Tripathy SJ, Padmanabhan K, Gerkin RC, Urban NN: Intermediate

- intrinsic diversity enhances neural population coding. Proc Natl Acad Sci U S A 2013, 110:8248-8253.

This is a theoretical study based on earlier work from the same group that found a diversity of biophysical response properties of mitral cells in the olfactory bulb, seeking to quantify the level of diversity that optimizes stimulus representation. The authors build populations of models based on the experimentally observed diversity using generalized linear models and Bayesian stimulus decoding to determine how effectively different populations encode a common stimulus. Interestingly, they find the optimal level of diversity is sufficient to eliminate some level of redundancy by ensuring decorrelation of neuronal response, while keeping some redundancy to ensure robust stimulus coding in the presence of external noise or damage - and the mitral cells seem to balance the benefits of diversity and feature similarity.

73. Padmanabhan $\mathrm{K}$, Urban NN: Disrupting information coding via

-. block of 4-AP-sensitive potassium channels. J Neurophysiol 2014, 112:1054-1066.

This study builds on previous work from the same group that identified intrinsic biophysical diversity in the responses of mitral cells in the olfactory bulb and demonstrated its implications for neural coding. The authors manipulate a specific ion channel, 4-amino-pyridine (4-AP)-sensitive potassium, and demonstrate that the level of biophysical diversity is directly correlated to the expression of this channel. In fact, adding 4-AP decreases stimulus information due to reduction in the diversity of population spike patterns. This study powerfully demonstrates that the coding capacity of a neuronal population can be affected by changes in a single ion channel.

74. Gjorgjieva J, Sompolinsky H, Meister M: Benefits of pathway splitting in sensory coding. J Neurosci 2014, 34:12127-12144. This study addresses the question of why information transmission is maximized when sensory signals are processed by multiple pathways (here focusing on ON, and OFF), typically implemented by different types of sensory neurons with diverse intrinsic properties. The authors build abstract models at the level of input-output relations (binary or sigmoidal 
nonlinearities) and ask for the optimal parameters to encode a onedimensional stimulus most efficiently. While the different models and stimulus conditions demonstrate individual differences in the specifics of the predicted cellular properties, the study finds that the highest improvement in performance occurs for stimulus distributions that are sparse - as observed in many instances in nature.

75. Kastner DB, Baccus SA, Sharpee TO: Critical and maximally informative encoding between neural populations in the retina. Proc Natl Acad Sci U S A 2015, 112:2533-2538.

76. Markram H, Muller E, Ramaswamy S, Reimann MW, Abdellah M, Sanchez CA, Ailamaki A, Alonso-Nanclares L, Antille N, Arsever S et al.: Reconstruction and simulation of neocortical microcircuitry. Cell 2015, 163:456-492.

77. Morgan RJ, Santhakumar amd V, Soltesz I: Modeling the dentate gyrus. Prog Brain Res 2007, 163:639-658.

78. Lee SH, Marchionni I, Bezaire M, Varga C, Danielson N, LovettBarron M, Losonczy A, Soltesz I: Parvalbumin-positive basket cells differentiate among hippocampal pyramidal cells. Neuron 2014, 82:1129-1244.

79. Wu J-Y, Tsau Y, Hopp H-P, Cohen LB, Tang AC, Falk CX: Consistency in nervous systems: trial-to-trial and animal-toanimal variations in the responses to repeated applications of a sensory stimulus in Apysia. J Neurosci 1994, 14:1366-1384.

80. Wu J-Y, Cohen LB, Falk CX: Neuronal activity during different behavior in Aplysia: a distributed organization. Science 1994, 263:820-823

81. Zochowski M, Cohen LB, Fuhrmann G, Kleinfeld D: Distributed and partially separate pools of neurons are correlated with two different components of the gill-withdrawal reflex in Aplysia. J Neurosci 2000, 20:8485-8492.

82. Biron D, Wasserman S, Thomas JH, Samuel AD, Sengupta P: An olfactory neuron responds stochastically to temperature and modulates Caenorhabditis elegans thermotactic behavior. Proc Natl Acad Sci U S A 2008, 105:11002-11007.

83. Larsch J, Ventimiglia D, Bargmann Cl, Albrecht DR: Global brain

-. dynamics embed the motor command sequence of Caenorhabditis elegans. Proc Natl Acad Sci U S A 2013 110:E4266-E4273.

This study develops techniques to image neuronal calcium activity from at least 20 Caenorhabditis elegans animals while simultaneously delivering precise chemical stimulation. The authors characterized responses of sensory neurons and interneurons to multiple odors, odor concentrations and temporal patterns. By doing so in freely moving animals, they were able to correlate neural recordings with behavior. They found significant variability in the behavioral responses, despite reproducible sensory neuron responses.

84. Gutierrez GJ, O’Leary T, Marder E: Multiple mechanisms switch - $\quad$ an electrically coupled, synaptically inhibited neuron between competing rhythmic oscillators. Neuron 2013, 77:845-858.

The authors examine several degenerate mechanisms by which a hub neuron can switch its participation in different sunbnetworks that oscillate at different frequencies. The study focuses on a five-cell subcircuit motivated by two networks in the stomatogastric ganglion that generate a fast and a slow rhythm. The switching of patterns in the network is examined as a function of the electrical and chemical inhibitory connections using a novel visualization technique called the 'parameterscape.'

85. Gutierrez GJ, Marder E: Modulation of a single neuron has state-dependent actions on circuit dynamics. eNeuro 2014, 1:E0009.

86. Fenton AA, Muller RU: Place cell discharge is extremely variable during individual passes of the rat through the firing field. PrOC Natl Acad Sci U S A 1998, 95:3182-3187.
87. Dombeck DA, Harvey CDD, Tian L, Looger LL, Tank DW: Functional imaging of hippocampal place cells at cellular resolution during virtual navigation. Nat Neurosci 2010, 13:1433-1440.

88. Baldassarre A, Lewis CM, Committeri G, Snyder AZ, Romani GL, Corbetta M: Individual variability in functional connectivity predicts performance of a perceptual task. Proc Natl Acad Sci U S A 2012, 109:3516-3521.

89. Mueller S, Wang D, Fox MD, Yeo BTT, Sepulcre J, Sabuncu MR

- $\quad$ Shafee R, Lu J, Liu H: Individual variability in functional connectivity architecture of the human brain. Neuron 2013, 77:586-595.

The authors use repeated-measurement resting-state functional MR to explore intersubject variability in connectivity. They find that individual differences in functional connectivity were nonuniformly distributed across the cortex, with different amounts of variability in different cortical regions. By comparing intersubject variability to maps of evolutionary cortical expansion, anatomical variability, long-range integration and regional segregation the authors search for a relationship to previously observed individual differences in cognition and behavior.

90. McGinley MJ, Vinck M, Reimer J, Batista-Brito R, Zagha E, Cadwell CR, Tolias AS, Cardin JA, McCormick DA: Waking state: rapid variations modulate neural and behavioral responses. Neuron 2015, 87:1143-1161.

91. Tripodi M, Evers J-F, Mauss A, Bate M, Landgraf M: Structural homeostasis: compensatory adjustments of dendritic arbor geometry in response to variations of synaptic input. PLOS Biol 2008, 6:e260.

92. Srikanth S, Narayanan R: Variability in state-dependent

- plasticity of intrinsic properties during cell-autonomous selfregulation of calcium homeostasis in hippocampal model neurons. eNeuro 2015, 2 e0053-15.2015.

This modeling study addresses the robustness of homeostatic tuning during switches in afferent input to a single neuron. While calcium-based homeostatic rules can reliably tune the intrinsic conductances of a single hippocampal neuron, tuning in the presence of functionally external input generates variable intrinsic properties and firing responses. The variability in tuning increases even further when two types of afferent input are provided to the neuron - and can sometimes even fail to produce functional output. This highlights the importance of state-dependent switches in afferent inputs on intrinsic properties during neural coding and homeostasis.

93. Gjorgjieva J, Clopath C, Audet J, Pfister J-P: A triplet spiketiming-dependent plasticity model generalizes the Bienenstock-Cooper-Munro rule to higher-order spatiotemporal correlations. Proc Natl Acad Sci U S A 2011, 108:19383-19388.

94. Anirudhan A, Narayanan R: Analogous synaptic plasticity - $\quad$ profiles emerge from disparate channel combinations. $J$ Neurosci 2015, 35:4691-4705.

This study uses computational approaches to determine the biophysical substrate of a specific plasticity rule, the Bienenstock-CooperMunro (BCM) rule, used for the modification of synaptic strength. The $\mathrm{BCM}$ rule has a sliding threshold that determines the activity set point at which synapses will be potentiated or depressed. The authors examine data-bases of hippocampal neurons with seven different ion channels and use sensitivity analysis to determine the set of channels that have the most substantial impact on regulating the sliding threshold of the BCM rule.

95. Connor J, Stevens C: Prediction of repetitive firing behavior from voltage clamp data on an isolated neuron soma. J Physiol 1971, 213:31-54. 